\title{
OPEN Synaptotagmin 7 switches short-term synaptic plasticity from depression to facilitation by suppressing synaptic transmission
}

\author{
Takaaki Fujii ${ }^{1}$, Akira Sakurai ${ }^{1}$, J. Troy Littleton ${ }^{2} \&$ Motojiro Yoshiharaa $^{1 凶}$
}

Short-term synaptic plasticity is a fast and robust modification in neuronal presynaptic output that can enhance release strength to drive facilitation or diminish it to promote depression. The mechanisms that determine whether neurons display short-term facilitation or depression are still unclear. Here we show that the $\mathrm{Ca}^{2+}$-binding protein Synaptotagmin 7 (Syt7) determines the sign of short-term synaptic plasticity by controlling the initial probability of synaptic vesicle (SV) fusion. Electrophysiological analysis of Syt7 null mutants at Drosophila embryonic neuromuscular junctions demonstrate loss of the protein converts the normally observed synaptic facilitation response during repetitive stimulation into synaptic depression. In contrast, overexpression of Syt7 dramatically enhanced the magnitude of short-term facilitation. These changes in short-term plasticity were mirrored by corresponding alterations in the initial evoked response, with SV release probability enhanced in Syt7 mutants and suppressed following Syt7 overexpression. Indeed, Syt7 mutants were able to display facilitation in lower $\left[\mathrm{Ca}^{2+}\right]$ where release was reduced. These data suggest $\mathrm{Syt} 7$ does not act by directly sensing residual $\mathrm{Ca}^{2+}$ and argues for the existence of a distinct $\mathrm{Ca}^{2+}$ sensor beyond Syt7 that mediates facilitation. Instead, Syt7 normally suppresses synaptic transmission to maintain an output range where facilitation is available to the neuron.

Synaptotagmins (Syts) are a large family of $\mathrm{Ca}^{2+}$ binding proteins, with the Syt 1 isoform functioning as the major $\mathrm{Ca}^{2+}$ sensor for synchronous synaptic vesicle (SV) fusion ${ }^{1} . \mathrm{Ca}^{2+}$ also controls presynaptic forms of short-term plasticity, with other Syt isoforms representing promising candidates to mediate these processes. Indeed, Synaptotagmin 7 (Syt7) has been reported to function in facilitation ${ }^{2}$, a form of short-term plasticity that enhances synaptic transmission following consecutive action potentials. Facilitation is believed to be mediated by residual $\mathrm{Ca}^{2+}$ acting to enhance the number of SVs that are released during repetitive action potentials occurring within a short temporal window ${ }^{3}$. Since Syt7 binds $\mathrm{Ca}^{2+}$ with high affinity ${ }^{4}$ and slow kinetics ${ }^{5}$, which match requirements for facilitation, the protein has been hypothesized to act as the $\mathrm{Ca}^{2+}$ sensor for this form of presynaptic plasticity. However, the role of Syt7 in facilitation is still unclear.

Drosophila neuromuscular junctions (NMJs) provide an excellent system for testing the role of Syt7 in shortterm synaptic plasticity. In particular, embryonic NMJs are highly plastic ${ }^{6}$ and allow stable recordings in high $\mathrm{Ca}^{2+}$ concentrations using the myosin heavy chain (Mhc) mutant background to prevent muscle contraction ${ }^{7}$ Together with the lack of compensation that might occur at older synapses, these advantages provide highly reliable measurements of synaptic transmission. Indeed, analysis of Syt1 mutants at embryonic NMJs established this Syt isoform functions as the synchronous $\mathrm{Ca}^{2+}$ sensor for synaptic transmission ${ }^{8-10}$. Although NMJs in many other species show depression, Drosophila embryonic NMJs are facilitative at physiological $\mathrm{Ca}^{2+}$ concentrations as shown here, similar to many mammalian central synapses such as those in the hippocampus ${ }^{11}$. Using stable recordings from this facilitative and plastic synapse in Drosophila embryos, we quantified the absolute value of synaptic currents in Syt7 mutants and in animals overexpressing Syt7. While loss of Syt7 enhanced presynaptic output, overexpression of Syt7 suppressed release. These changes in the magnitude of presynaptic output were

${ }^{1}$ Memory Neurobiology Project, National Institute of Information and Communications Technology, Kobe, Japan. ${ }^{2}$ The Picower Institute for Learning and Memory, Department of Biology, Department of Brain and Cognitive Sciences, Massachusetts Institute of Technology, Cambridge, MA, USA. ${ }^{\square}$ email: motojiro@nict.go.jp 
mirrored by changes in short-term presynaptic plasticity. High levels of Syt7 enabled robust facilitative responses while loss of Syt7 switched the normally facilitating synapse into one that displayed short-term depression. This work reveals that Syt7 normally reduces synaptic transmission to scale it to an appropriate range where facilitation is allowed, providing a bi-directional switch for short-term synaptic plasticity.

\section{Results}

Syt7 switches short-term synaptic plasticity from depression to facilitation. Whole-cell voltage clamp recordings were performed at muscle fiber 6 in Drosophila embryos at hatching stage (21-24 h after fertilization) to record synaptic currents (EPSCs) elicited by stimulation of the glutamatergic motoneurons innervating the muscle. All recordings were done in the background of a null mutation in Myosin heavy chain $\left(M h c^{1}\right)$ to prevent muscle contraction ${ }^{7}$. We stimulated the nerve for 10 pulses at $10 \mathrm{~Hz}$, commonly used in studies of both mammalian central synapses and Drosophila NMJs for mimicking natural communication ${ }^{11}$, at the reported physiological concentration of $\mathrm{Ca}^{2+}(1.5 \mathrm{mM})^{12}$. As shown in Fig. 1a,b, wild type (WT) embryos display a cumulating increase in response to three consecutive stimuli at the beginning of the $10 \mathrm{~Hz}$ stimulation, while Syt7 null homozygous mutant $\left(S y t 7^{-/-}\right)^{13}$ embryos show a decreasing response from the larger 1st EPSC than that of WT. The response then reaches a plateau until presumed depletion of the immediate releasable SV pool (IRP) occurs ${ }^{14}$. Strikingly, heterozygotes $\left(S y t 7^{+/-}\right)$containing only a single copy of Syt7 showed an intermediate phenotype with almost no facilitation or depression, consistent with the intermediate size of the 1st EPSC compared to WT or Syt7 $7^{--}$(Fig. 1a,b). The ratio of the 3rd EPSC to 1st EPSC in Fig. 1c demonstrates switching from depression to facilitation with increasing amounts of Syt7. These results suggest Syt7 normally suppresses synaptic transmission to an appropriate range to prevent depletion of the IRP and ensure synaptic facilitation. To test this hypothesis directly, we overexpressed Syt7 with the elav-GAL4 pan-neuronal driver. Syt7 overexpression suppressed the amplitude of the 1st EPSC compared to WT (Fig. 1a,b) and increased the facilitation ratio by more than sixfold compared with elav-GAL4 controls (Fig. 1d). These results indicate the levels of Syt7 set the initial magnitude of presynaptic output, with normal levels of Syt7 suppressing synaptic transmission to a range where facilitation can occur.

Syt7 null mutants show enhanced nerve-evoked transmission with higher release probability than WT. We next analyzed individual EPSCs from WT and Syt7 $7^{-/}$. As shown in Fig. 2a,b, at all $\mathrm{Ca}^{2+}$ concentrations tested $S y t 7^{-/-}$mutants show dramatically enhanced synaptic currents that are three-fold larger than WT at $0.5 \mathrm{mM} \mathrm{Ca}^{2+}$. Presynaptic changes that can drive larger synaptic responses can be secondary to increased release probability $(P)$ of single SV fusion events or an increased number $(N)$ of readily-releasable SVs. To differentiate between these two possibilities, we measured the readily-releasable pool size using hypertonic

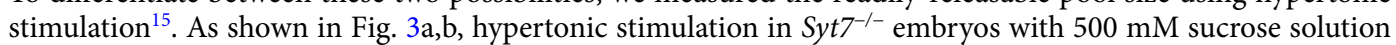
induced SV release levels similar to WT, indicating mutants do not have a larger releasable SV pool at embryonic synapses. Although the SV pool size is different in Syt7 3rd instar mutants ${ }^{13}$, SV pools increase dramatically from embryonic development through the 3rd instar larval stage as synaptic maturation and profound synaptic growth occur. Given the dramatic increase in presynaptic release in embryonic Syt7 terminals can occur without a change in the releasable SV pool size at this stage of development, release probability for individual SVs is also enhanced following loss of Syt7.

We previously found that $S y t 1^{-/-}$null mutants have a smaller releasable SV pool than $\mathrm{WT}^{8,9}$. We assayed for genetic interactions in SV pool size between $S y t 1^{-/-}$and $S y t 7^{-/-}$and found that loss of Syt7 did not change the smaller response in Syt1 $1^{-/-}$mutants (Fig. 3a,b), consistent with enhanced synaptic transmission occurring without an elevated SV pool size in $S y t 7^{-/-}$. We next measured quantal size generated by release of single SVs during hypertonic stimulation, where release is $\mathrm{Ca}^{2+}$-independent and does not show multi-quantal release ${ }^{16}$. Quantal sizes of WT and $S y t 1^{-/-}$are similar to those previously found ${ }^{8}$, and both $S y t 7^{-/-}$and $S y t 1^{-/-}$; Syt $7^{-/-}$double mutants did not alter quantal size (Fig. 3c). These findings indicate there is no defect in the postsynaptic response to SV release in the absence of Syt7.

These data indicate the stronger synaptic transmission in $S y t 7^{-/-}$mutants is likely due to increased $P$ rather than changes in $N$. It was previously proposed that Syt1 triggers SV fusion upon action potential-induced $\mathrm{Ca}^{2+}$ influx and also clamps fusion at lower $\left[\mathrm{Ca}^{2+}\right]$ to generate a better signal-to-noise ratio ${ }^{17}$. Experimental evidence supporting the clamping model were recorded at Drosophila 3rd instar NMJs where miniature frequency in Syt $1^{-/-}$is increased compared to $\mathrm{WT}^{18,19}$. In the case of embryonic NMJs, we did not detect an elevated frequency of miniature events in $S y t 1^{-/-8,9}$. However, $S y t 7^{-/-}$mutants displayed more frequent miniature release than WT, and $S y t 1^{-/}$; $S y t 7^{-/-}$double mutants showed even more frequent miniature release than $S y t 7^{-/-}$single mutants (Fig. 4a,b). These results support a model where both Syt7 and Syt1 may clamp SV fusion in a synergistic manner as observed at mammalian synapses ${ }^{20}$. We hypothesize that elevated miniature release rate in Syt $^{-/-}$single mutant embryos is cancelled out by the reduced SV pool size at these synapses, as well as its reported role in SV docking and endocytosis ${ }^{8,9}$.

Syt7 suppresses release probability and enables paired-pulse facilitation. The higher release probability in $S y t 7^{-/-}$mutants predicts decreased facilitation would occur, given synapses with stronger release probability have a lower paired pulse facilitation (PPF) ratio due to depletion of SVs during the 1st response $\mathrm{e}^{3,11}$. Indeed, the PPF ratio in $S y t 7^{-/-}$was much smaller than WT at $0.5 \mathrm{mM}$ and $1.5 \mathrm{mM}$ extracellular [Ca ${ }^{2+}$ (Fig. 5a,b). However, at a lower $\left[\mathrm{Ca}^{2+}\right]$ of $0.3 \mathrm{mM}$, a positive PPF ratio was observed in $S y t 7^{-/-}$in accordance with the smaller amplitude of the 1st EPSC (Fig. 5a,b). As shown in Fig. 5c, Syt $7^{-/-}$shows a relatively high PPF ratio (arrowhead in Fig. 5c) when synaptic currents are small, similar to that observed in WT. Together with the enhanced facili- 
a

Overexpression of Syt 7 (Syt7 OE)

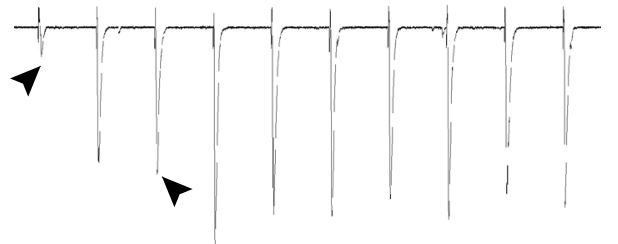

WT

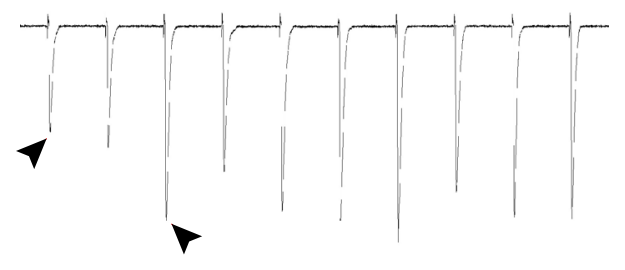

Syt7 $7^{+/-}$
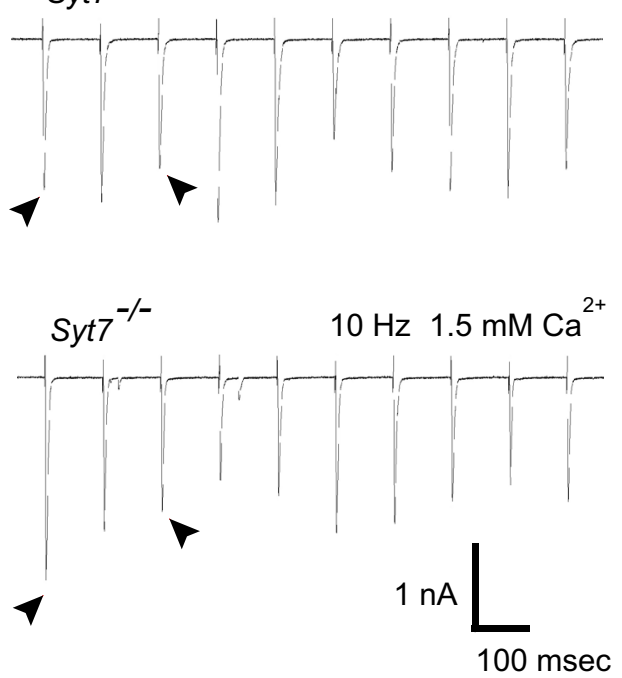

b

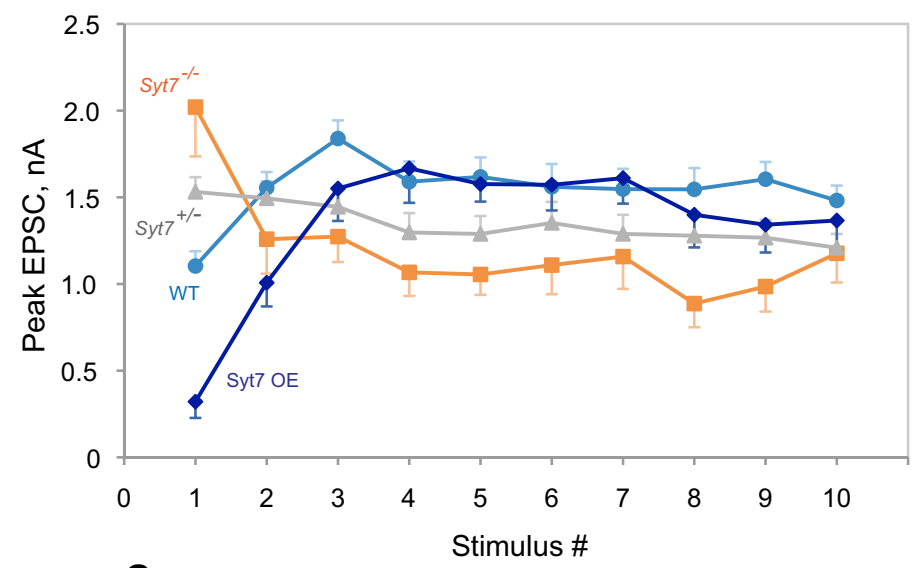

C

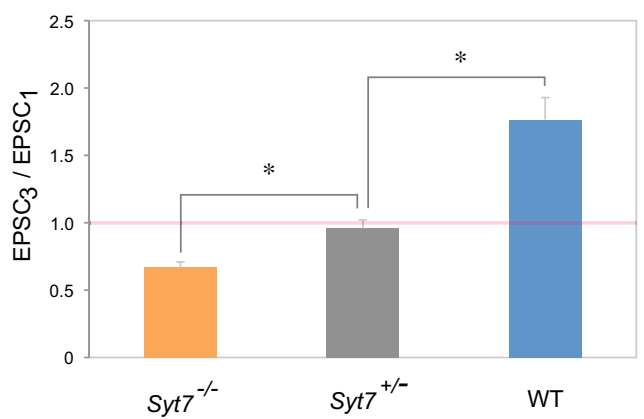

d

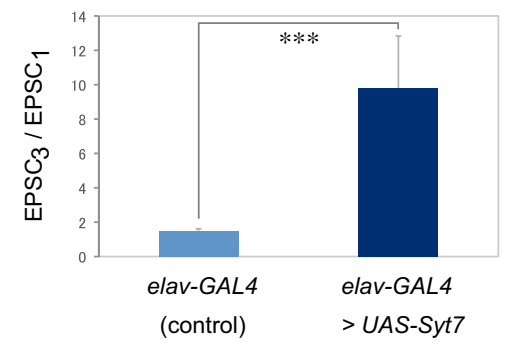

(Syt7 OE)

Figure 1. Syt7 switches short-term plasticity from depression to facilitation. (a) Representative traces of synaptic currents in response to 10 pulses of $10 \mathrm{~Hz}$ repetitive stimulation at $1.5 \mathrm{mM} \mathrm{Ca}^{2+}$ in embryos

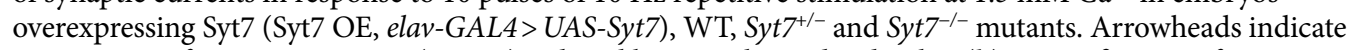
comparison of synaptic currents (EPSCs) induced by 1st pulse and 3rd pulse. (b) Quantification of $10 \mathrm{~Hz}$ stimulation experiments. The number of recorded cells analyzed for each genotype: Syt7 OE, 9; WT, 13; Syt7 ${ }^{+/-}$, 13; $S y t 7^{-/-}, 13$. (c) Facilitation or depression ratios shown as $3 \mathrm{rd}$ pulse-induced EPSC $\left(\mathrm{EPSC}_{3}\right) / 1$ st pulse-induced EPSC $\left(\right.$ EPSC $_{1}$ ). WT, Syt7 $7^{+-}$and $S y t 7^{-/-}$mutants were analyzed with the Kruskal-Wallis test using a one-way ANOVA by ranks and significant difference between the groups was found $(\mathrm{P}<0.0001) .{ }^{\star} \mathrm{P}<0.05$ by Dunn's post-hoc multiple comparison test between groups. The red line indicates when no facilitation or depression is observed $($ ratio $=1)$. (d) Facilitation ratio shown as 3rd pulse-induced EPSC $\left(\mathrm{EPSC}_{3}\right) / 1$ st pulse-induced EPSC (EPSC $)_{1}$ ) for elav-GAL4 controls and Syt7 OE (elav-GAL4>UAS-Syt7). The results were analyzed with the Mann-Whitney $U$ test and significant difference between the groups was found $\left({ }^{* *} \mathrm{P}<0.001\right)$. The numbers of recorded cells analyzed for each genotype: elav-GAL4, 13; elav-GAL4>UAS-Syt7, 9. Error bars are SEM.

tation observed following Syt7 overexpression (Fig. 1d), these data indicate Syt7 levels bi-directionally gate the sign of short-term plasticity (facilitation versus depression) by controlling the levels of presynaptic output.

\section{Discussion}

The current study indicates Syt7 is indispensable for facilitation across the physiological range of $\mathrm{Ca}^{2+}$ concentrations at Drosophila embryonic NMJs as previously shown for mammalian preparations ${ }^{2}$. In the absence of Syt7, the normally facilitating embryonic NMJ now displays depression. Following Syt7 overexpression, facilitation is 


$$
0.5 \mathrm{mM} \mathrm{Ca}^{2+}
$$

a

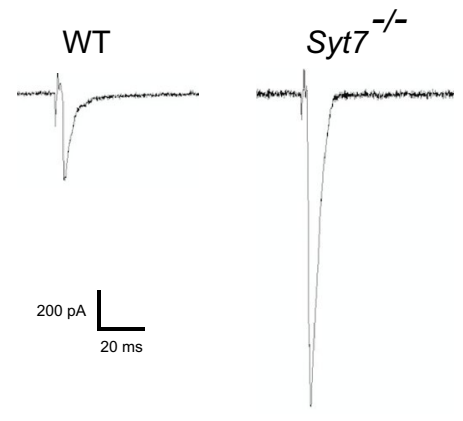

b

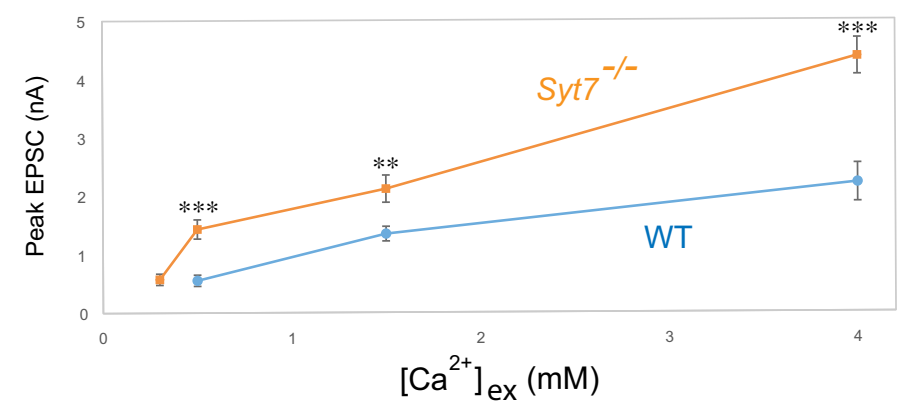

Figure 2. Syt7 null mutants show enhanced nerve-evoked transmission. (a) Representative traces of evoked synaptic currents in wild type (WT) and $S y t 7^{-/-}$at $0.5 \mathrm{mM} \mathrm{Ca}^{2+}$ and $1.5 \mathrm{mM} \mathrm{Ca}^{2+}$. (b) $\mathrm{Ca}^{2+}$ dependence of peak evoked synaptic currents (EPSC). WT and Syt $7^{-/-}$were analyzed at each $\mathrm{Ca}^{2+}$ concentration with Student's $t$ test, and significant difference between the two groups was found at $0.5 \mathrm{mM} \mathrm{Ca}^{2+}(* * \mathrm{P}<0.001), 1.5 \mathrm{mM} \mathrm{Ca}^{2+}$ $\left({ }^{*} \mathrm{P}<0.01\right)$ and $\left.4 \mathrm{mM} \mathrm{Ca}^{2+}{ }^{* * *} \mathrm{P}<0.001\right)$. WT was not quantified at $0.3 \mathrm{mM} \mathrm{Ca}^{2+}$ due to the high probability of failures. The numbers of recorded cells analyzed for each genotype: (WT) $0.5 \mathrm{mM}, 11 ; 1.5 \mathrm{mM}, 11 ; 4 \mathrm{mM}, 8$ $\left(\right.$ Syt $\left.7^{-/}\right) 0.3 \mathrm{mM}, 19 ; 0.5 \mathrm{mM}, 15 ; 1.5 \mathrm{mM}, 20 ; 4 \mathrm{mM}, 9$. Error bars are SEM.

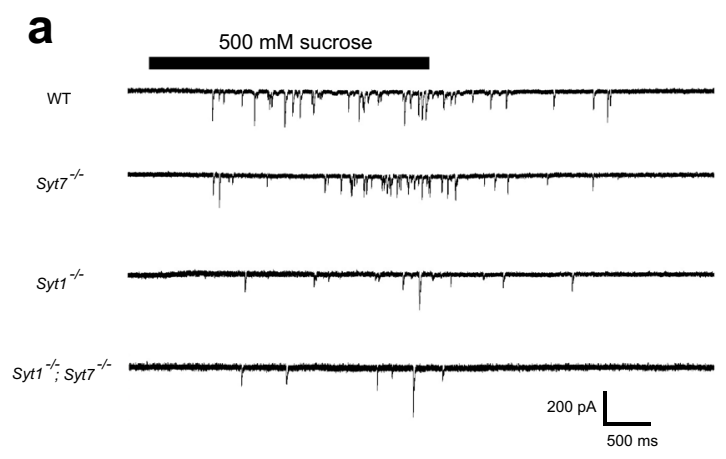

b

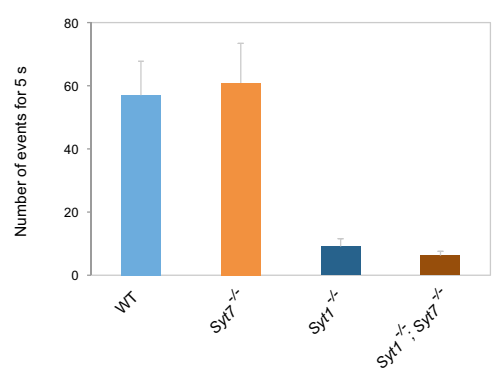

C

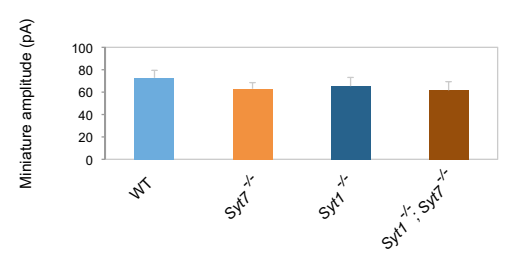

Figure 3. Hypertonic-induced miniature release. (a) Representative traces for each genotype. $500 \mathrm{mM}$ sucrose was applied for $3 \mathrm{~s}$ (bar) in the absence of $\mathrm{Ca}^{2+}$. (b) Number of hypertonic-induced miniature synaptic currents recorded during $5 \mathrm{~s}$ after the beginning of application of $500 \mathrm{mM}$ sucrose. Numbers of recorded cells: WT, 24; Syt7 $7^{-/}, 24 ;$ Syt1 $1^{-/-}, 38 ; S y t 1^{-/-} ;$Syt7 $7^{-/-}, 39$. (c) Amplitude of miniature synaptic currents induced by hypertonic stimulation with $500 \mathrm{mM}$ sucrose. Amplitudes recorded from a single muscle cell were averaged and the averages for each cell are shown. Number of recorded cells are the same as those in b. Error bars are SEM. 
a

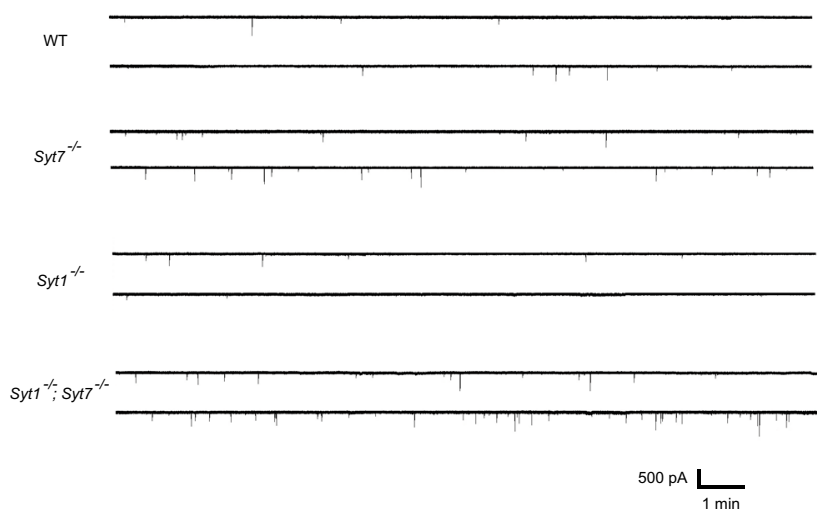

b

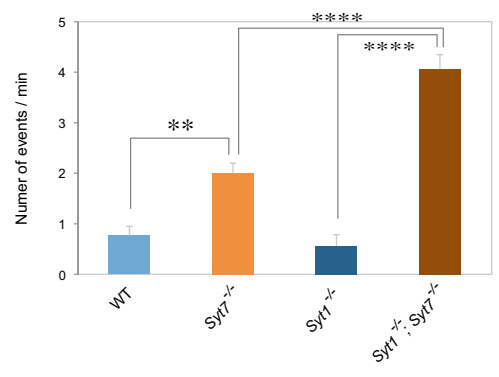

Figure 4. Spontaneous miniature synaptic currents. (a) Representative traces for each genotype in the presence of $3 \mu \mathrm{M}$ Tetrodotoxin (TTX), which inhibits action potentials. Concentration of $\mathrm{Ca}^{2+}$ was $1.5 \mathrm{mM}$. (b) Miniature synaptic currents counted during $10 \mathrm{~min}$ and shown as number of events per minute for each genotype. The four groups were analyzed with ordinary one-way ANOVA and significant difference between the groups was found $(\mathrm{P}<0.0001) .{ }^{* *} \mathrm{P}<0.01,{ }^{* * * *} \mathrm{P}<0.0001$ by Tukey's post-hoc multiple comparison test between groups. The

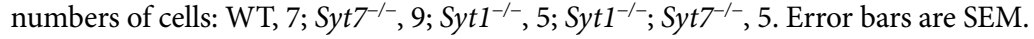

greatly enhanced. Our data indicate the major reason for defective facilitation in $S y t 7^{-/-}$mutants is due to loss of Syt7's ability to suppress release, which likely causes rapid SV depletion that is non-compatible with short-term synaptic facilitation. Likewise, overexpression of Syt7 reduces SV release and allows for enhanced facilitation. This role for Syt7 contrasts with current models proposed in mammals where Syt7 is hypothesized to bind residual $\mathrm{Ca}^{2+}$ to directly act as a facilitation $\mathrm{Ca}^{2+}$ sensor. Although our data indicate Syt7 is not the primary $\mathrm{Ca}^{2+}$ sensor for facilitation, we cannot rule out the possibility that Syt7 has dual roles in both suppressing and facilitating SV fusion as observed for Syt $1^{9,21}$. If Syt7 has a dual role with C2A functioning for clamping and C2B for facilitation, the null mutant would lack both properties. It is possible the lack of clamping is the dominant phenotype, with any facilitative function being masked by SV depletion at higher $\mathrm{Ca}^{2+}$ concentrations. Thus, we cannot rule out a Syt7-dependent component of facilitation." However, the presence of facilitation in Syt $7^{-/-}$mutants at lower $\left[\mathrm{Ca}^{2+}\right]$ (Fig. 5) indicate there is a facilitation sensor besides Syt7 that monitors residual $\mathrm{Ca}^{2+}$ to directly activate this form of short-term plasticity. Although we cannot completely rule out a role for residual maternally supplied Syt7 at the embryonic stage in Syt $7^{-/-}$mutants, there is no evidence from RNA profiling studies (Flybase) that indicate Syt7 is present at earlier stages of embryonic development prior to nervous system formation. Thus, it is unlikely residual Syt7 could sustain normal levels of facilitation as observed in low $\left[\mathrm{Ca}^{2+}\right]$, consistent with the enhanced synaptic transmission observed across a broad $\left[\mathrm{Ca}^{2+}\right]$ range in $\mathrm{Syt} 7^{-/-}$mutants. Given facilitation is also present in low $\left[\mathrm{Ca}^{2+}\right]$ in $S y t 7^{-/-}$mutants at the 3rd instar stage when any maternal contribution would be depleted $^{13}$, we conclude that facilitation can occur in the complete absence of Syt7 under conditions where the initial response is reduced.

A key advantage of the Drosophila embryonic NMJ preparation is the ability to unambiguously monitor the absolute baseline values of synaptic strength even in high $\left[\mathrm{Ca}^{2+}\right]$ using the non-contracting Mhc mutant. In this regard, it is clear that synaptic transmission at Drosophila embryonic NMJs is much stronger in Syt $7^{-/-}$mutants at all $\mathrm{Ca}^{2+}$ concentrations tested. Moreover, the stronger transmission is due to higher release probability rather than an increased number of releasable SVs. Thus, our data predict that higher release probability leads to a lower facilitation ratio secondary to vesicle depletion ${ }^{3,11}$. The precise mechanisms by which Syt7 suppresses SV release to enable facilitation will require further study. Beyond a potential clamping function for Syt7, the protein could alter local $\mathrm{Ca}^{2+}$ buffering or cause increased $\mathrm{Ca}^{2+}$ influx that could contribute to elevated SV release. Syt7 does not localize to SVs and may instead act from the plasma membrane ${ }^{22}$ or internal membrane compartments ${ }^{13}$, allowing for several potential mechanisms for Syt7 to suppress release. $\mathrm{Ca}^{2+}$ binding to the $\mathrm{C} 2 \mathrm{~A}$ and $\mathrm{C} 2 \mathrm{~B}$ domains of Syt 1 have been shown to have distinct functions in SV release, with C2B playing a dominant role in triggering $\mathrm{SV}$ fusion and C2A acting to clamp release ${ }^{9,23}$. It is unclear if the C2A and C2B domains of Syt7 act similarly in Drosophila or have independent functions compared to Syt1. One possibility is that the C2A domain of Syt7 suppresses SV fusion and the C2B domain facilitates release, similar to Syt1. Structure function studies of Syt7 should help elucidate this biology in Drosophila, similar to our prior studies of Syt 1 function.

As suppression of SV release by Syt7 is dose-dependent ${ }^{13}$ (Fig. 1b), increasing levels of Syt7 would elevate the ratio of facilitation as shown in Fig. 1a-d. These results suggest the degree of facilitation across distinct neuronal populations may be set by Syt7 levels similar to a potentiometer. Our analysis of $S y t 7^{-/-}$nulls, heterozygotes and overexpression lines support such a model that changes release and short-term plasticity in a graded fashion. Depending on whether a synapse is facilitative or depressive ${ }^{11}$, Syt7 expression could be modulated to gate plasticity to the level that most benefits the local circuit, similar to how Syt1 and Syt2 levels variably control release synchronicity across neuronal populations ${ }^{20}$. Indeed, the squid giant synapse is facilitative only when $\mathrm{Ca}^{2+}$ is lowered from normal saline (artificial sea water) ${ }^{24,25}$, similar to Syt $7^{-/-}$mutants, suggesting synapses that normally depress may have reduced levels of Syt7. Indeed, recent evidence suggests that species-specific differences in presynaptic plasticity in rodents is linked to the levels of Syt $7^{26}$. In shrews, the levels of Syt7 are lower in 

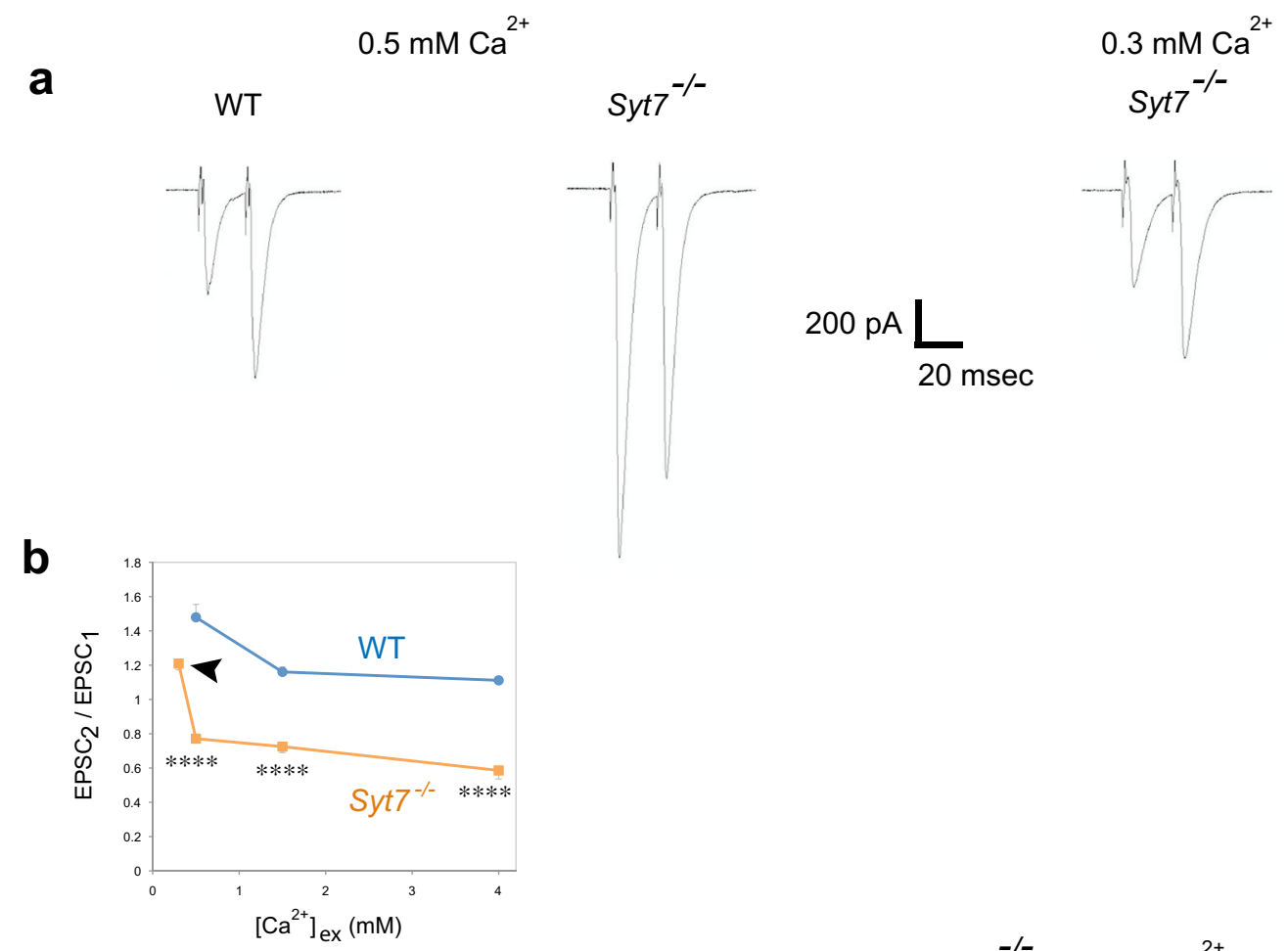

C
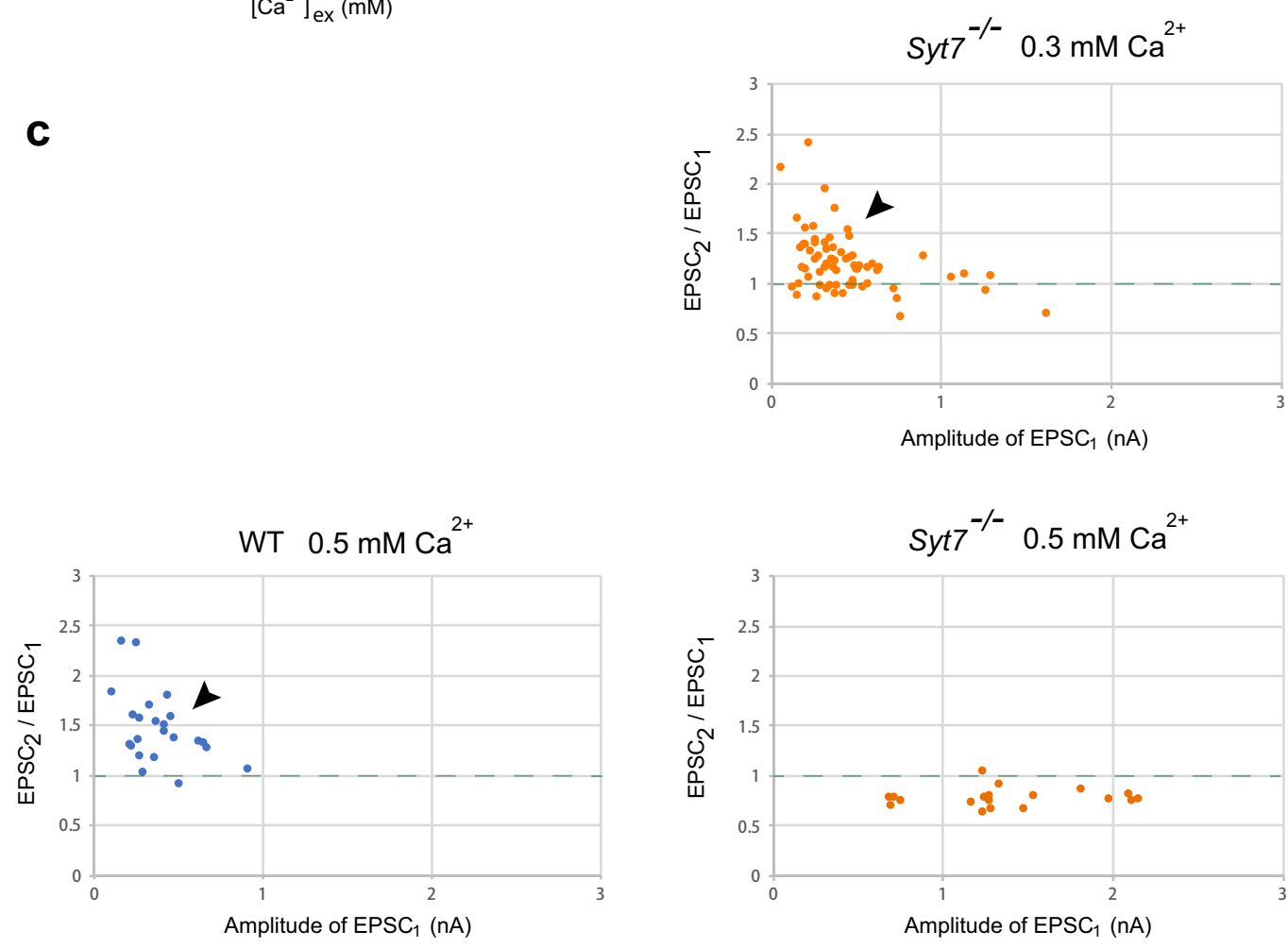

Figure 5. Syt7 suppresses release probability and enables paired-pulse facilitation. (a) Representative traces of paired-pulse facilitation with $20 \mathrm{~ms}$ intervals in WT and $S y t 7^{-/-}$at $0.5 \mathrm{mM} \mathrm{Ca}^{2+}$ and in $S y t 7^{-/-}$at $0.3 \mathrm{mM}$ $\mathrm{Ca}^{2+}$. Twenty sets of responses to paired pulses were averaged. (b) $\mathrm{Ca}^{2+}$ dependence of facilitation ratio as $2 \mathrm{nd}$ pulse-induced EPSC $\left(\mathrm{EPSC}_{2}\right) / 1$ st pulse-induced EPSC $\left(\mathrm{EPSC}_{1}\right)$ in WT and $S y t 7^{-l_{-}}$. Note facilitation at $0.3 \mathrm{mM}$ $\mathrm{Ca}^{2+}$ in $S y t 7^{-/-}$(arrowhead). WT and Syt $7^{-/-}$were analyzed at each $\mathrm{Ca}^{2+}$ concentration with Mann-Whitney $U$ test, and significant difference between the two groups was found at $0.5 \mathrm{mM} \mathrm{Ca}^{2+}\left({ }^{* * * *} \mathrm{P}<0.0001\right), 1.5 \mathrm{mM}$ $\mathrm{Ca}^{2+}\left({ }^{* * *} \mathrm{P}<0.0001\right)$ and $4 \mathrm{mM} \mathrm{Ca}^{2+}\left({ }^{* * * *} \mathrm{P}<0.0001\right)$. Ratio at $0.3 \mathrm{mM} \mathrm{Ca}^{2+}$ in WT was not quantified due to the high probability of failures in response to the 1st pulse. The numbers of samples analyzed for each genotype: (WT) $0.5 \mathrm{mM}, 23 ; 1.5 \mathrm{mM}, 10 ; 4 \mathrm{mM}, 10\left(\right.$ Syt7 $\left.^{-/}\right) 0.3 \mathrm{mM}, 68 ; 0.5 \mathrm{mM}, 19 ; 1.5 \mathrm{mM}, 11 ; 4 \mathrm{mM}$, 13. (c) Ratios of $\mathrm{EPSC}_{2} / \mathrm{EPSC}_{1}$ at $0.3 \mathrm{mM}$ (only Syt7 ${ }^{-/}$) and $0.5 \mathrm{mM}$. Responses in (b) were plotted with amplitude of $\mathrm{EPSC}_{1}$ along the X-axis. Note facilitation observed when $\mathrm{EPSC}_{1}$ is small (arrowheads). Error bars are SEM. 
hippocampal CA3 synapses and they show reduced presynaptic plasticity. In contrast, Syt7 levels are much higher in mice, with their CA3 output synapses displaying far greater forms of presynaptic plasticity. Drosophila adults ${ }^{27}$ and 3rd instar larvae ${ }^{28}$ also have less facilitative NMJs than embryonic NMJs. This difference may contribute to the distinct effects of Syt7 on clamping spontaneous SV release that is observed between embryonic and 3rd instar NMJs ${ }^{13}$. Mammalian studies identified redundant functions for Syt1 and Syt7 in clamping spontaneous fusion at inhibitory synapses ${ }^{29}$. While reductions in Syt7 levels alone did not increase spontaneous SV release, removal of both Syt1 and Syt7 enhanced mini frequency to a far greater level that loss of Syt1 alone. In addition, a Syt7 transgene was able to rescue the elevated miniature frequency in Syt 1 mutants. These differences in clamping properties were attributed to an insufficient level of Syt7 expression compared to Syt1. Differences in the Syt1/Syt7 ratio between Drosophila 3rd instar and embryonic NMJs may also contribute to distinct effects on spontaneous SV clamping observed in Syt1 and Syt7 mutants at these distinct developmental stages. In conclusion, controlling expression level of Syt7 provides an attractive mechanism for activity-dependent presynaptic scaling of release probability as a homeostat for both presynaptic output and short-term facilitation, similar to postsynaptic scaling mechanisms previously described for chronic forms of synaptic plasticity ${ }^{30}$.

\section{Methods}

Drosophila strains. Drosophila melanogaster were cultured on standard medium at $25^{\circ} \mathrm{C}$. The $S y t 7^{\mathrm{M} 2}$ null mutant was used in this study ${ }^{13}$. Syt $7^{M 2}$ has an insertion of a Minos transposon into the second exon of the Syt7 that generates a premature stop codon before the C2A domain, resulting in loss of Syt7 protein by Western analysis ${ }^{13}$. We observed similar results for $S y t 7^{M 1}$, an independent null mutant generated via CRISPR-Cas 9 (data not shown). All electrophysiological recordings were carried out in the background of a null mutant of muscle-specific myosin heavy chain $\left(M h c^{1}\right)$ as previously described ${ }^{7}$. The $M h c^{1}$ mutant had no observed effect on synapse formation, neurotransmitter release, or postsynaptic glutamate receptor clustering ${ }^{7,8}$. Syt 1 null mutants were generated by crossing Syt1 $1^{N 13}$, an intragenic Syt1 deficiency ${ }^{31}$, with Syt1 ${ }^{A D 4}$, which truncates Syt1 before the transmembrane domain ${ }^{19}$. These null alleles were recombined with a chromosome containing $M h c^{1}$. Mutant second chromosomes carrying $M h c^{1}$ or both $M h c^{1}$ and $S y t 1$ alleles were placed over a $C y O$ balancer containing Actin-driven GFP or Dfd-driven GFP (Bloomington Stock Center) to allow unambiguous identification of embryos with Syt1 null and $M h c^{1}$ backgrounds. UAS transgenes were expressed using a GAL4 driver under control of the pan-neuronal elav promoter ${ }^{32}$ on the third chromosome. All lines carried the $w$-background to allow recognition of the $\left[w^{+}\right]$marker in transgenic strains. Syt7 overexpression UAS lines have been previously described $^{13}$.

Electrophysiological analysis. All electrophysiology experiments were carried out as described previously ${ }^{8,9}$. Briefly, synaptic currents were recorded with the patch-clamp technique in whole-cell configuration from embryonic muscle fiber 6 at segments A2-A4 that were maintained at a holding potential of $-60 \mathrm{mV}$. Embryos were aged 21-24 h after fertilization and recorded in HL3.1 saline solution ${ }^{33}$ (in mM: $\mathrm{NaCl}, 70 ; \mathrm{KCl}, 5$; $\mathrm{MgCl}_{2}, 5.5 ; \mathrm{NaHCO}_{3}, 10$; trehalose, 5; sucrose, 115; Hepes-NaOH, 5; pH 7.2), using an Multiclamp 700B amplifier (Axon Instrument/Molecular Device) at $23-25^{\circ} \mathrm{C}$. External saline solutions with various concentrations of $\mathrm{Ca}^{2+}$ were prepared by replacing $\mathrm{MgCl}_{2}$ with $\mathrm{CaCl}_{2}$. The internal solution in patch pipettes contained: (in mM) $\mathrm{KCl}, 155$; ATP, 2; EGTA, 5; HEPES-KOH, 10, pH 7.1. Before recording, embryos were dissected in $\mathrm{Ca}^{2+}$-free HL3.1 and treated for $1 \mathrm{~min}$ with $0.35 \mathrm{mg} / \mathrm{mL}$ collagenase (type IV; Sigma) in $0.1 \mathrm{mM} \mathrm{Ca}^{2+}$ saline solution. For nerve stimulation, a small part of an intact (uncut) motor nerve was sucked into a suction electrode containing bath solution at its site of emergence from the CNS, and $1.5 \mu \mathrm{A}$ of positive current was delivered for $1 \mathrm{~ms}$. For extensive electrical isolation, a negative glass electrode with a blunt end was loosely attached to the nerve at a more distal site than that of the suction electrode. Clampex 10.7 in the pCLAMP10.7 package (Molecular Devices) was used for data acquisition and data were analyzed with Clampfit 10.7 in the pCLAMP10.7 (Molecular Devices).

For hypertonic-stimulated release, $500 \mathrm{mM}$ sucrose dissolved in $\mathrm{Ca}^{2+}$-free HL-3.1 saline solution was included in a puff pipette with a $1-\mu \mathrm{m}$ tip. The pipette was placed in close vicinity of the boundary between muscle fibers 6 and 7 where the NMJ forms. Hypertonic solution was puffed using positive pressure for $3 \mathrm{~s}$. Slow responses originating from electrically coupled muscle fibers were excluded in subsequent analysis in all genotypes. These experiments were performed in $\mathrm{Ca}^{2+}$-free saline to avoid enhancements of presynaptic release mediated by retrograde signaling downstream of postsynaptic $\mathrm{Ca}^{2+}$ influx $^{6,34}$. Miniature analysis was performed with $1.5 \mathrm{mM} \mathrm{Ca}^{2+}$ HL3.1 containing $3 \mu \mathrm{M}$ tetrodotoxin (TTX). Through the "Gap free" recording of Clampex, miniature release was counted for $10 \mathrm{~min}$ for each cell and reported as a frequency per minute.

Statistical analysis was performed using Prism6 software (GraphPad). Since the variation of data between animals or cells was small compared with the larger variation in data from one cell or one animal, results were combined and the number of events recorded are shown as $\mathrm{N}$.

Received: 12 June 2020; Accepted: 22 January 2021

Published online: 18 February 2021

\section{References}

1. Yoshihara, M., Adolfsen, B. \& Littleton, J. T. Is synaptotagmin the calcium sensor?. Curr. Opin. Neurobiol. 13, 315-323 (2003).

2. Jackman, S. L., Turecek, J., Belinsky, J. E. \& Regehr, W. G. The calcium sensor synaptotagmin 7 is required for synaptic facilitation. Nature 529, 88-91 (2016).

3. Zucker, R. S. \& Regehr, W. G. Short-term synaptic plasticity. Annu. Rev. Physiol. 64, 355-405 (2002).

4. Wang, P., Chicka, M. C., Bhalla, A., Richards, D. A. \& Chapman, E. R. Synaptotagmin VII is targeted to secretory organelles in PC12 cells, where it functions as a high-affinity calcium sensor. Mol. Cell Biol. 25, 8693-8702 (2005). 
5. Hui, E. et al. Three distinct kinetic groupings of the synaptotagmin family: Candidate sensors for rapid and delayed exocytosis. Proc. Natl. Acad. Sci. U.S.A. 102, 5210-5214 (2005).

6. Yoshihara, M., Adolfsen, B., Galle, K. T. \& Littleton, J. T. Retrograde signaling by Syt 4 induces presynaptic release and synapsespecific growth. Science 310, 858-863 (2005).

7. Yoshihara, M., Suzuki, K. \& Kidokoro, Y. Two independent pathways mediated by cAMP and protein kinase A enhance spontaneous transmitter release at Drosophila neuromuscular junctions. J. Neurosci. 20, 8315-8322 (2000).

8. Yoshihara, M. \& Littleton, J. T. Synaptotagmin I functions as a calcium sensor to synchronize neurotransmitter release. Neuron 36, 897-908 (2002).

9. Yoshihara, M., Guan, Z. \& Littleton, J. T. Differential regulation of synchronous versus asynchronous neurotransmitter release by the C2 domains of synaptotagmin 1. Proc. Natl. Acad. Sci. U.S.A. 107, 14869-14874 (2010).

10. Mackler, J. M., Drummond, J. A., Loewen, C. A., Robinson, I. M. \& Reist, N. E. The $\mathrm{C}_{2} \mathrm{~B} \mathrm{Ca}^{2+}$-binding motif of synaptotagmin is required for synaptic transmission in vivo. Nature 418, 340-344 (2002).

11. Dobrunz, L. E. \& Stevens, C. F. Heterogeneity of release probability, facilitation, and depletion at central synapses. Neuron 18, 995-1008 (1997).

12. Stewart, B. A., Atwood, H. L., Renger, J. J., Wang, J. \& Wu, C. F. Improved stability of Drosophila larval neuromuscular preparations in haemolymph-like physiological solutions. J. Comp. Physiol. A 175, 179-191. https://doi.org/10.1007/BF00215114 (1994).

13. Guan, Z., Quinones-Frias, M. C., Akbergenova, Y. \& Littleton, J. T. Drosophila Synaptotagmin 7 negatively regulates synaptic vesicle release and replenishment in a dosage-dependent manner. eLife 9, e55443 (2020).

14. Delgado, R., Maureira, C., Oliva, C., Kidokoro, Y. \& Labarca, P. Size of vesicle pools, rates of mobilization, and recycling at neuromuscular synapses of a Drosophila mutant, shibire. Neuron 28, 941-953 (2000).

15. Rosenmund, C. \& Stevens, C. F. Definition of the readily releasable pool of vesicles at hippocampal synapses. Neuron 16, 1197-1207 (1996).

16. Koenig, J. H., Yamaoka, K. \& Ikeda, K. Calcium-induced translocation of synaptic vesicles to the active site. J. Neurosci. 13, 2313-2322 (1993)

17. Popov, S. V. \& Poo, M. M. Synaptotagmin: A calcium-sensitive inhibitor of exocytosis?. Cell 73, 1247-1249 (1993).

18. Littleton, J. T., Stern, M., Schulze, K., Perin, M. \& Bellen, H. J. Mutational analysis of Drosophila synaptotagmin demonstrates its essential role in $\mathrm{Ca}(2+)$-activated neurotransmitter release. Cell 74, 1125-1134 (1993).

19. DiAntonio, A. \& Schwarz, T. L. The effect on synaptic physiology of synaptotagmin mutations in Drosophila. Neuron 12, 909-920 (1994).

20. Turecek, J. \& Regehr, W. G. Neuronal regulation of fast synaptotagmin isoforms controls the relative contributions of synchronous and asynchronous release. Neuron 101, 938-949 (2019).

21. Nishiki, T. \& Augustine, G. J. Synaptotagmin I synchronizes transmitter release in mouse hippocampal neurons. J. Neurosci. 24, 6127-6132 (2004).

22. Sugita, S. et al. Synaptotagmin VII as a plasma membrane $\mathrm{Ca}^{2+}$ sensor in exocytosis. Neuron 30, 459-473 (2001).

23. Nishiki, T. I. \& Augustine, G. J. Calcium-dependent neurotransmitter release: Synaptotagmin to the rescue. J. Comp. Neurol. 436, 1-3 (2001).

24. Charlton, M. P. \& Bittner, G. D. Presynaptic potentials and facilitation of transmitter release in the squid giant synapse. J. Gen. Physiol. 72, 487-511 (1978).

25. Stanley, E. F. Decline in calcium cooperativity as the basis of facilitation at the squid giant synapse. J. Neurosci. 6, 782-789 (1986).

26. Beed, P. et al. Species-specific differences in synaptic transmission and plasticity. Sci. Rep. 10, 16557 (2020).

27. Kawasaki, F., Hazen, M. \& Ordway, R. W. Fast synaptic fatigue in shibire mutants reveals a rapid requirement for dynamin in synaptic vesicle membrane trafficking. Nat. Neurosci. 3, 859-860 (2000).

28. Renger, J. J., Ueda, A., Atwood, H. L., Govind, C. K. \& Wu, C. F. Role of cAMP cascade in synaptic stability and plasticity: Ultrastructural and physiological analyses of individual synaptic boutons in Drosophila memory mutants. J. Neurosci. 20, 3980-3992 (2000).

29. Bacaj, T. et al. Synaptotagmin-1 and synaptotagmin-7 trigger synchronous and asynchronous phases of neurotransmitter release. Neuron 80, 947-959 (2013).

30. Turrigiano, G. G., Leslie, K. R., Desai, N. S., Rutherford, L. C. \& Nelson, S. B. Activity-dependent scaling of quantal amplitude in neocortical neurons. Nature 391, 892-896 (1998).

31. Littleton, J. T., Stern, M., Perin, M. \& Bellen, H. J. Calcium dependence of neurotransmitter release and rate of spontaneous vesicle fusions are altered in Drosophila synaptotagmin mutants. Proc. Natl. Acad. Sci. U.S.A. 91, 10888-10892 (1994).

32. Campos, A. R., Rosen, D. R., Robinow, S. N. \& White, K. Molecular analysis of the locus elav in Drosophila melanogaster: A gene whose embryonic expression is neural specific. EMBO J. 6, 425-431 (1987).

33. Feng, Y., Ueda, A. \& Wu, C. F. A modified minimal hemolymph-like solution, HL3.1, for physiological recordings at the neuromuscular junctions of normal and mutant Drosophila larvae. J. Neurogenet. 18, 377-402 (2004).

34. Malgaroli, A. \& Tsien, R. W. Glutamate-induced long-term potentiation of the frequency of miniature synaptic currents in cultured hippocampal neurons. Nature 357, 134-139 (1992).

\section{Acknowledgements}

This work was supported by JSPS KAKENHI Grant Numbers JP26891030, JP19H00998 and JP19H04767 to M.Y.; JP16K18375 and JP19K16275 to A.S. and NIH grant NS40296 to J.T.L. We thank the Bloomington Drosophila Stock Center for fly strains, and Daisuke Yamamoto and members of the Yoshihara lab for helpful discussions and comments on manuscript. We thank Kazuhiro Oiwa, Hiroaki Kojima, and Iwao Hosako for generous support at Kobe.

\section{Author contributions}

M.Y. conceived and designed the experiments, discussing with A.S., T. F. and J.T.L. A.S. carried out genetic manipulations. J.T.L. provided genetic information and the Syt7 null mutant. T.F. and M.Y. collected electrophysiology data and analyzed the data. M.Y. interpreted the data and wrote the manuscript with input from the other authors.

\section{Competing interests}

The authors declare no competing interests.

\section{Additional information}

Correspondence and requests for materials should be addressed to M.Y.

Reprints and permissions information is available at www.nature.com/reprints. 
Publisher's note Springer Nature remains neutral with regard to jurisdictional claims in published maps and institutional affiliations.

(c) (i) Open Access This article is licensed under a Creative Commons Attribution 4.0 International License, which permits use, sharing, adaptation, distribution and reproduction in any medium or format, as long as you give appropriate credit to the original author(s) and the source, provide a link to the Creative Commons licence, and indicate if changes were made. The images or other third party material in this article are included in the article's Creative Commons licence, unless indicated otherwise in a credit line to the material. If material is not included in the article's Creative Commons licence and your intended use is not permitted by statutory regulation or exceeds the permitted use, you will need to obtain permission directly from the copyright holder. To view a copy of this licence, visit http://creativecommons.org/licenses/by/4.0/.

(C) The Author(s) 2021 